\title{
Prozessmerkmale guten Unterrichts mit Flipped Classroom
}

\author{
Didaktische Ansprüche an Homeschooling
}

Heidemarie Ulbrich ${ }^{1}$

https://doi.org/10.53349/resource.2021.i16.a1003

\section{Zusammenfassung}

Chancen und Herausforderungen im Homeschooling, besonders in diesem digitalen Zeitalter, gab es im letzten Jahr mehr als genug. Guter Unterricht wird in Zeiten von Schullockdowns im Rahmen der COVID-19-Pandemie von den Pädagog*innen mehr denn je gefordert. Die Merkmale guten Unterrichts wie etwa jene von Meyer rücken also wieder stärker in den Fokus. Unterrichts- und Lernkonzepte unter Einsatz digitaler Medien mit den passenden Unterrichtsmethoden bedürfen einer Neuorientierung durch die Pädagog*innen. Der Lehrrahmen von Bloom als Grundgerüst für weitere Unterrichtskonstrukte sowie dessen Top-Down-Ansicht in der Unterrichtsmethode „Flipped Classroom“ sind wichtige Merkmale für einen guten Unterricht im Homeschooling.

$\begin{array}{ll}\text { Schlüsselwörter: } & \text { Keywords: } \\ \text { Unterrichtsqualität } & \text { Teaching quality } \\ \text { Corona-Unterricht } & \text { Corona-Lesson } \\ \text { Unterrichtsmethoden } & \text { Teaching practice }\end{array}$

\section{Einleitung - Lehren und Lernen im digitalen Zeitalter}

Medien sind ein zentraler Bestandteil der heutigen Lebenswelt eines Kindes bzw. eines Jugendlichen. „Aus Sicht der SchülerInnen bringt der Einsatz elektronischer Medien vor allem eines: Spaß und Abwechslung“ (Mayr, Resinger \& Schratz, 2009, S. 37). Synergieeffekte aus Digitalisierung und Bildung gehören zum heutigen Schulbetrieb. Die COVID-19-Pandemie zwang die Schulen in der Sekundarstufe I und II, ihren Unterricht neu zu organisieren, zu planen und zu strukturieren. Trotzdem verstärkt Meyer (2004) in seinem Beitrag „Didaktische Ansprüche an Homeschooling und Fernunterricht“, dass die Mehrzahl der veröffentlichten Kriterienkataloge wie jener von Helmke (2012) sowie sein eigener (Meyer, 2004) ihre Gültigkeit behalten, mehr noch, sie sind noch wichtiger geworden (Meyer, 2020).

Daher bieten diverse Lernkonzepte wie z. B. die Methode Flipped Classroom eine Basis, um Schüler*innen mit ihren Arbeitsmitteln (Smartphone, Tablet oder Laptop) Lernstoff näher zu bringen und anschließend im Unterricht zu festigen. Aus reinem E-Learning wurde rasch Blended Learning, d. h. einerseits sind die Lehr- und Lernkonzepte im Fokus, andererseits die passende Verwendung digitaler Medien. Reinmann (2005) bezeichnet Blended Learning als konsensfähige Etikette für Lehr- und Lernkonzepte, die digitale Medien als festen Bestandteil von Lernumgebungen betrachten und den Fokus gezielt auf den Mehrwert digitaler Medien für die Optimierung sowie Erweiterung von Präsenzlernen legen. Weiters sind auch verschiedene Methoden und Organisationsformen des Lernens zu kombinieren (Reinmann, 2005, S. 11).

Konnte die COVID-19-Krise von Lehrpersonen als Chance genutzt werden, um mit digitalen Mitteln ihren Unterricht sinnvoller zu gestalten?

\footnotetext{
${ }^{1}$ Pädagogische Hochschule Oberösterreich, Kaplanhofstraße 40, 4020 Linz

E-Mail: heidemarie.ulbrich@ph-ooe.at
} 
Erste Befunde des Schul-Barometers in Deutschland, Österreich und der Schweiz liefern Daten zu digitalen LehrLern-Formaten und den Erfahrungen und Empfehlungen aus der ersten Welle. Aus der Befragung geht hervor, dass die Schulschließungen die Mehrheit der Mitarbeitenden der Schule zumindest teilweise vor Herausforderungen bzgl. digitaler Lehr-Lern-Formen stellen. Lediglich 15 Prozent erlebten den ersten Lockdown eher nicht als Herausforderung. Natürlich kann in den Daten des Schul-Barometers gezeigt werden, dass die Lehrer*innen unterschiedliche Erfahrungen bezüglich ihrer fortgeschrittenen Professionalität in der Digitalisierung aufweisen (Huber, Günther, Schneider, Helm, Schwander, Schneider \& Pruitt, 2020, S. 24).

Eine der Herausforderungen von Homeschooling ist die Adaption der Kriterien für guten Unterricht. Aus diesem Grund wird im Rahmen dieses Beitrags der Frage nachgegangen, welche Merkmale guten Unterrichts für das Homeschooling gelten und inwieweit diese im Rahmen der Methode des Flipped Classrooms berücksichtigt werden können. Dazu werden im nächsten Unterkapitel die „traditionellen“ Prozessmerkmale guten Unterrichts dargestellt. Anschließend werden die Prozessmerkmale guten Unterrichts im Homeschooling beschrieben und anhand der Methode des Flipped Classrooms konkreter spezifiziert. Abschließend wird die Forschungsfrage zusammengefasst beantwortet und ein Ausblick auf weitere Forschungsfelder gegeben.

\section{Prozessmerkmale guten Unterrichts}

„Es kommt auf den*die Lehrer*in an!“ Nicht selten werden diese oder so ähnliche Aussagen über guten Unterricht getroffen. Eine auf empirischen Daten basierende Pädagogik müsste eigentlich die Frage folgendermaßen stellen: „Wie kommt es, dass der Unterricht nicht ist, was er zu sein beanspruchen muss und was er sein könnte, und was trägt der Lehrer dazu bei?“ (Gruschka, 2007, S. 11)

Eine Reihe von pädagogischen Psycholog*innen sowie Bildungswissenschaftler*innen sieht es als ihre Aufgabe, die Antworten auf die oben gestellte Frage zu verbreiten. Ging es in den 80ern um die "gute Schule“, geht es in diesen Jahren um „guten Unterricht“. Der Pädagoge Meyer (2015) geht der Frage in seinem Buch zum guten Unterricht nach. Helmke (2015), der pädagogische Psychologe, beantwortet sie implizit in seinem weitverbreiteten Buch „Unterrichtsqualität und Lehrerprofessionalität - Diagnose, Evaluation und Verbesserung des Unterrichts". In seinem Vierfelderschema stellt der Autor prozess- vs. produktorientierte Sichtweisen der Unterrichtsqualität dar (Helmke, 2015, S. 23).

Spitzer (2011) formulierte es zur Person der Lehrerin*des Lehrers so: „Keine Technik ist gut genug, solange Therapeut und Klient nicht miteinander klarkommen. Sonst findet kein Umlernen, keine Neuorientierung und vor allem keine Heilung statt. Die Therapie verläuft positiv, wenn sie einander schätzen und mögen." Er stellt somit fest, ob ein*e Lehrer*in am Computer oder der Tafel unterrichtet, ob Frontalunterricht oder Gruppenarbeiten durchgeführt werden, ob mono- oder dialogisch gearbeitet wird, ist egal: „Wichtig ist zunächst einmal, ob sich Lehrende und Schüler gegenseitig schätzen und mögen“ (Spitzer 2011, S. 412). Spitzers wissenschaftliche Arbeit sollte jedoch hinterfragt werden. Dennoch, auch Hattie $(2014$, S. 21) zieht die Schlussfolgerung, dass die Lehrperson ein wichtiger Faktor für den Lernerfolg darstellt: „Lehrpersonen gehören zu den wirkungsvollsten Einflüssen beim Lernen“ (Hattie, 2014, S. 21).

Guter Unterricht stellt aber nicht nur die Frage nach dem Verhältnis von "gut" (Adjektiv) und „Unterricht" (Substantiv), sondern differenziert guten von schlechtem Unterricht. Gruschka (2007) stellt in seiner Betrachtung der Forschungsfrage von Meyer wie auch Helmke eine Unklarheit in der Betrachtung der Alltäglichkeit des Unterrichts mit einer nicht zureichenden Deskription von allerlei Faktoren sowie eine doppelsinnige Normalität dar, die als widersprüchliche Gleichzeitigkeit von Norm und empirisch Gegebenem dient. Der Lerneffekt wird als Maßstab gesetzt und soll klar definieren, welche Faktoren sie befördern bzw. behindern (Gruschka, 2007, S. 13).

\section{Meyers Zehn Merkmale}

Die Zehn Merkmale von Meyer werden aus der Unterrichtsforschung und vorwiegend aus dem Klassiker „Mastery Learning“ (Bloom, 1968), Techniken der Klassenführung (Kounin, 2006), Fünfzehntausend Stunden (Rutter, 1980) (Gruschka, 2007, S. 16) gezogen. Besonders korrespondiert die Arbeit mit den Überblickswerken 
zur Unterrichtsforschung (das amerikanische Werk von Brophy (2000) und die deutsche Übersicht von Helmke (2003)).

Mit Hilfe von Theoretiker*innen sowie Praktiker*innen stellt Meyer (2017, S. 17) seine folgenden 10 Merkmale zusammen:

1. Klare Strukturierung des Unterrichts (Prozess-, Ziel- und Inhaltsklarheit; Rollenklarheit, Absprache von Regeln, Ritualen und Freiräumen)

2. Hoher Anteil echter Lernzeit (durch gutes Zeitmanagement, Pünktlichkeit; Auslagerung von Organisationskram; Rhythmisierung des Tagesablaufs

3. Lernförderliches Klima (durch gegenseitigen Respekt, verlässlich eingehaltene Regeln, Verantwortungsübernahme, Gerechtigkeit und Fürsorge)

4. Inhaltliche Klarheit (durch Verständlichkeit der Aufgabenstellung, Plausibilität des thematischen Gangs, Klarheit und Verbindlichkeit der Ergebnissicherung)

5. Sinnstiftendes Kommunizieren (durch Planungsbeteiligung, Gesprächskultur, Sinnkonferenzen, Lerntagebücher und Schülerfeedback)

6. Methodenvielfalt (Reichtum an Inszenierungstechniken; Vielfalt der Handlungsmuster; Variabilität der Verlaufsformen und Ausbalancierung der methodischen Großformen)

7. Individuelles Fördern (durch Freiräume, Geduld und Zeit; durch innere Differenzierung und Integration; durch individuelle Lernstandsanalysen und abgestimmte Förderpläne; besondere Förderung von Schüler*innen aus Risikogruppen)

8. Intelligentes Üben (durch Bewusstmachen von Lernstrategien, passgenaue Übungsaufträge, gezielte Hilfestellungen und „überfreundliche“ Rahmenbedingungen)

9. Transparente Leistungserwartungen (durch ein an den Richtlinien oder Bildungsstandards orientiertes, dem Leistungsvermögen der Schüler*innen entsprechendes Lernangebot und zügige förderorientierte Rückmeldungen zum Lernfortschritt)

10. Vorbereitete Umgebung (durch gute Ordnung, funktionale Einrichtung und brauchbares Lernwerkzeug)

Betrachtet man diese Merkmale aus Sicht des Homeschoolings, so zeigt sich, dass diese Merkmale trotz veränderter Rahmenbedingungen ihre Relevanz behalten. Teilweise gewinnen einzelne Merkmale gegenüber dem Präsenzunterricht an Bedeutung. Die Situation auf Distanz etwa fordert eine noch klarere und transparentere Kommunikation der Struktur und der Regeln im Onlineunterricht, da hier Informationen leichter überhört werden, und auch Nachfragen ist im Vergleich zum Präsenzunterricht mit mehr Aufwand verbunden. In den ersten Befunden des Schul-Barometers in Deutschland, Österreich und der Schweiz bekunden einige Eltern und Lehrer*innen ihre eigene Überforderung aufgrund Überfrachtung mit zu vielen unterschiedlichen digitalen Lehr-Lern-Formen (Huber, Günther, Schneider, Helm, Schwander, Schneider \& Pruitt, 2020, S. 72). Besonders die Motivation und selbstständige Auseinandersetzung der Schüler*innen mit dem digitalen LehrLern-Format sind in den Augen der Lehrerschaft als auch der Schulleitungen problematisch, da ein Teil der Kinder Erklärungen und Lernbegleitung bräuchte. Die Unterstützung der Eltern bei digitalem Lernen wird hier noch mehr als beim Arbeiten mit Lehrmitteln und Arbeitsblättern benötigt (Huber, Günther, Schneider, Helm, Schwander, Schneider \& Pruitt, 2020, S. 62). Die sinnvolle Gestaltung von Arbeitsaufträgen in ihrer Klarheit und Struktur zu erstellen, ist hier umso wichtiger und rückt somit in den Mittelpunkt des Homeschoolings (Huber, Günther, Schneider, Helm, Schwander, Schneider \& Pruitt, 2020, S.70).

\section{Prozessmerkmale guten Unterrichts im Homeschooling}

Meyer (2020) definiert Prozessmerkmale guten Unterrichts für die spezielle Situation des Homeschoolings.

Dazu definiert er Homeschooling folgendermaßen:

Homeschooling ist ein durch die Schule organisierter Fernunterricht, in dem das gemeinsame Arbeiten in der Klasse/im Lernverband zeitlich befristet aufgehoben und durch individualisierte Hausarbeit ersetzt wird. Sie wird in unterschiedlichem Umfang von den Eltern bzw. Erziehungsberechtigten 
beaufsichtigt und von der Schule durch die Arbeit mit Bildungsservern und den Einsatz digitaler Medien unterstützt. (Meyer, 2020)

Meyer (2020) legt in seinem Beitrag insgesamt sechs didaktische Ansprüche an Homeschooling fest:

1. Das vor Beginn des Homeschooling aufgebaute Arbeitsbündnis nutzen:

Lehrpersonen und Schüler*innen brauchen für einen funktionierenden Unterricht eine Verantwortungsgemeinschaft, wobei sie sich gegenseitig beim Lehren und Lernen unterstützen.

2. Mit kognitiv und sozial aktivierenden Aufgabenstellungen arbeiten:

Innere Differenzierung durch in unterschiedlichen Leistungsniveaus formulierte Aufgabenstellungen ermöglichen, die nicht nur kognitiv, sondern auch sozial aktivierend sein sollen. Das fachdidaktische Know-how und die methodische Fantasie sind von den Lehrenden hier besonders gefragt.

3. Selbstreguliertes Lernen stärken:

Hier wird die Selbstbestimmungstheorie der Motivation (Deci \& Ryan, 2000) von Meyer hervorgehoben. Die Selbstbestimmungstheorie von Deci und Ryan geht davon aus, dass Menschen drei grundlegende, voneinander unabhängige Bedürfnisse haben, die in engem Zusammenhang zur intrinsischen und extrinsischen Motivation stehen. Neben den zwei Bedürfnissen nach Selbstbestimmung (Autonomie) und Kompetenzerleben (eigener Kompetenz, eigener Fähigkeit) postulieren Deci und Ryan ein drittes Bedürfnis, das vor allem bei extrinsischem Verhalten wirksam ist. Da extrinsisches Verhalten mit der daraus resultierenden Konsequenz eine wichtige Rolle spielt, ist das dritte Bedürfnis nach sozialer Eingebundenheit besonders wichtig. Personen wollen sich in das soziale Gefüge eingebunden fühlen und sind dadurch motiviert, zu handeln (Rustemeyer, 2011, S. 30).

4. Feedback nehmen und geben:

Meyer (2020) weist hier auf die formative Leistungsdiagnostik hin, die hohe Effektstärke von $d=0,73$ hat bereits Hattie in seiner Studie errechnet. Die Lernwirksamkeit geht verloren, sobald die Lehrperson versucht, in den Hintergrund zu treten. Wenn auch ein erheblicher Arbeitsaufwand durch die Rückmeldungen der Lehrperson - auch mit digitalen Medien gut möglich -, ist sie grundlegend wichtig für den Lernprozess.

5. Leistungsschwächere Schüler*innen bevorzugen:

Meyer (2020) geht davon aus, dass sich die negativen Wirkungen der sozialen Kopplung beim Homeschooling potenzieren werden, besonders für leseschwache Schüler*innen ist Homeschooling eine besondere Herausforderung.

6. Den Einsatz von Arbeitsblättern drosseln:

Durch die Flut an Arbeitsblättern, die auf die Schüler*innen einprasselt, wird schnell Langweile und wenig Herausforderndes bei der Bearbeitung empfunden.

Meyer (2020) stellt zusammenfassend fest, dass es für erfolgreiches, gelingendes und qualitativ umgesetztes Homeschooling einen guten Umgang mit digitalen Unterrichtsmedien und viel Engagement gekoppelt mit didaktisch-methodischer Phantasie braucht.

Die Relevanz dieser Ansprüche zeigt sich auch in aktuellen empirischen Befunden. So stellen etwa Huber und Helm (2020, S. 56) basierend auf Daten des Schul-Barometers in Deutschland, Österreich und der Schweiz fest, dass insbesondere Schüler*innen aus sozioökonomisch schlechter gestellten Familien Herausforderungen im Homeschooling erleben. Dabei scheint insbesondere das Fehlen der Kompetenz, selbstgesteuert zu lernen bzw. den Tag selbständig zu organisieren, einen negativen Einfluss auf die Lernerfahrungen im Homeschooling zu haben.

\subsection{Vielfalt der Lehr- und Lernmethoden im Homeschooling}

In der facheinschlägigen Literatur lässt sich eine Vielfalt an Unterrichtsmethoden finden. Wiechmann (1999, S. 17) nennt hier in einem Überblick Frontalunterricht, direktes Unterrichten, Gruppenpuzzle, Stationenarbeit, Wochenplanarbeit, entdeckendes Lernen und viele weitere. 
"Lehrmethoden sind bestimmte wiederkehrende Muster von Lehraktivitäten, die der Vermittlung von Lehrzielen und Lehrinhalten dienen, also Lernen bewirken sollen und von vielen Lehrern angewendet werden können" (Terhart, 1997, S. 24).

Die Lehrmethode umfasst einen großen Bereich von Dimensionen, die Terhart $(1997$, S. 25) folgendermaßen darstellt: kognitive Strukturierung des Unterrichts und soziale Strukturierung, sachstrukturelle Anordnung und andere mehr. Bei der Lernmethode geht es vor allem um äußere und innere Aktivitäten der Schüler*innen und darum, Handlungen zu verinnerlichen, Wissen einzuordnen, wie Auffassungstätigkeiten, Denkformen, Übungsformen, Kooperationsformen. Damit günstige Bedingungen für das Auslösen von Lernprozessen stattfinden, werden verschiedene Lehrmethoden eingesetzt. Lernen kann nur von der*dem Lernenden selbst vollzogen und nicht erzwungen werden (Terhart, 1997, S. 149).

Keine Methode ist prinzipiell besser als andere Methoden - es kommt immer auf den Kontext an. Dieser wird bestimmt durch die Klassenstufe, das Fach, den Inhalt, die zu erlernenden Kompetenzen, durch die Leistungsfähigkeit und -bereitschaft der Schüler*innen sowie die Methodenpräferenz und Persönlichkeit der Lehrperson. (Werner, Ebel, Spannagel \& Bayer, 2018, S. 14)

Weiters führt Spannagel an, dass die Methode Flipped Classroom nur für einige Inhalte passt und nicht angewendet werden soll, weil sie hip, neu oder modern ist (Werner, Ebel, Spannagel \& Bayer, S. 15). Die Ansicht, dass Flipped Classroom nicht die Lösung für alle schulischen Herausforderungen und Bildungsprobleme ist, teilen also Buchner und Schmid mit Spannagel. Auch sie führen an, dass jede Lehrperson für sich herauszufinden hat, ob der Flipped Classroom für ihre Klassen und für die Erreichung der Lernziele eine sinnvolle Methode ist (Buchner \& Schmid, 2019, S. 17).

\subsection{Taxonomie Blooms nach Anderson und Krathwohl}

Heutzutage ist die Bloomsche Taxonomie ein Klassiker. Bereits 1956 wurde von Benjamin Bloom u.a. diese Taxonomie herausgegeben (Baumgartner, 2011, S. 36), die schon in den fünfziger und sechziger Jahren des vorigen Jahrhunderts in den USA eine sehr häufig verwendete Taxonomie von Lernzielen war (Mayer, Hertnagel \& Weber, 2009, S. 32).

Ihr Grundmuster ist eine Liste von sechs kognitiven Bildungszielen, wovon jedes wiederum unterschiedliche Detailgrade aufweist. Die Taxonomie ist damit eindimensional aufgebaut. Darin besteht ein wesentlicher Unterschied zu ihrer zweidimensionalen Erweiterung in Form einer Tabelle durch Anderson und Kolleg*innen (Baumgartner, 2011, S. 35).

Die Bloomsche Taxonomie zeigte deutlich zwei ihrer Grenzen auf (Baumgartner, 2011, S. 36):

- Das Gliederungssystem geht auf die kognitiven Lehr- und Lernaspekte ein, sowohl der emotionale Bereich (Krathwohl, Bloom \& Masia, 1965) als auch die psychomotorischen Lernziele (Harrow, 1972) sind ausgeklammert. Diese wurden jedoch später durch eigene Ordnungssysteme ergänzt.

- Es gilt ein relativ hoher Abstraktionsgrad, wodurch sich der Vorteil ergibt, dass die Taxonomien auf alle Fachgebiete anwendbar sind.

Die Bloomschen Taxonomien zeichnen sich im Unterschied zu Gagné (US-amerikanischer experimenteller Psychologe und Pädagoge) und Ausubel (US-amerikanischer Pädagoge, Lerntheoretiker und Hochschullehrer) durch die Weite, sprich Berücksichtigung, der kognitiven, affektiven und psychomotorischen Dimensionen und ihre Anwendungsfreundlichkeit aus. Dies erklärt die große Verbreitung der Lernzieltaxonomien von Bloom (Mayer, Hertnagel \& Weber, 2009, S. 40)

Die Taxonomie gliedert sich in folgende drei Bereiche (Krathwohl, Bloom \& Masia, 1975, S. 6):

1. Kognitive Lernziele: Erinnern oder Reproduzieren eines Stoffes

2. Affektive Lernziele: Interessen, Einstellungen, Wertschätzungen, Werte oder emotionale Handlungen

3. Psychomotorische Lernziele: muskuläre oder motorische Fertigkeit im Umgang mit Material, Gegenständen, Handlung 
Die Lernzieltaxonomie von Bloom wurde von Arbeitsgruppen immer wieder weiterentwickelt. Ein Versuch führte zu Andersons-Taxonomietafel. Anderson und Krathwohl treffen eine klare Trennung und eine weitere Ausdifferenzierung von der Wissensdimension und Leistungsdimension, wobei sich diese Taxonomie auf kognitive Prozesse bezieht (Mayer, Hertnagel \& Weber, 2009, S. 41).

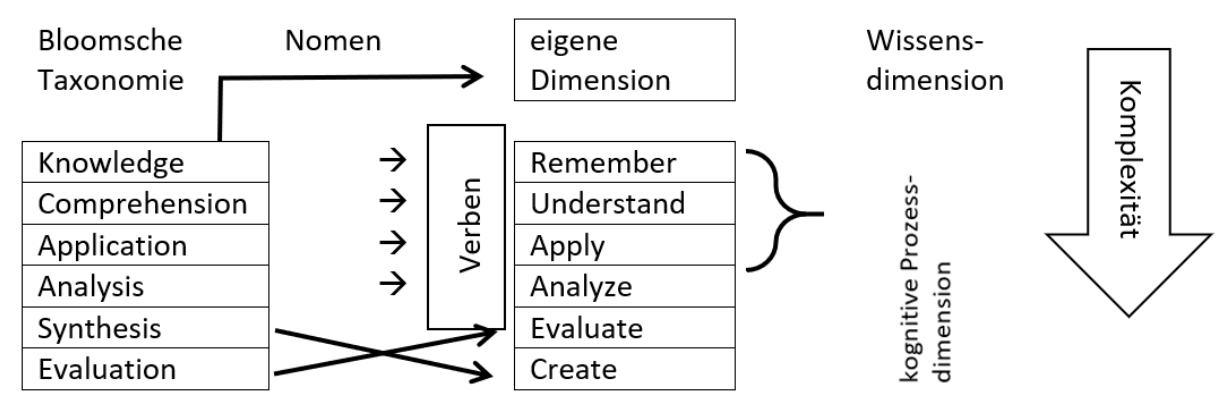

Abb. 1: Erweiterung der Bloomschen Taxonomie (eigene Darstellung nach Schöftner, 2018, S. 97)

„Die Leistungsdimensionen werden in Verbform beschrieben und die Wissensdimension („Knowledge dimension“) erhält zusätzliche (Unter-)Kategorien“ (Schöftner, 2018, S. 97). „Dadurch erhält diese Taxonomie eine genauere Planung in Bezug auf die Wissensdimensionen und hebt die Bedeutung von Prozesswissen sowie der metakognitiven Reflexion des Lernens hervor" (Mayer, Hertnagel \& Weber, 2009, S. 41). Die sechs kognitiven Lernzielkategorien (Erinnern, Verstehen, Anwenden, Analysieren, Bewerten und Schaffen) sind hierarchisch aufgebaut, wobei der Ansatz von Blooms Taxonomie sich nur in der Rangveränderung von Evaluieren und Erschaffen unterscheidet (Mayer, Hertnagel \& Weber, 2009, S. 43).

„Die Wissensdimensionen entsprechen einem Kontinuum vom Konkreten zum Abstrakten, wobei vier verschiedene Wissenstypen (Faktenwissen, Konzeptwissen, prozedurales Wissen sowie metakognitives Wissen) unterschieden werden“ (Mayer, Hertnagel \& Weber, 2009, S. 43). Anderson und Krathwohl kreierten somit eine Taxonomietafel mit 24 Zellen (Kombinationsmöglichkeiten) mit sechs kognitiven Lernzielkategorien und vier Wissensdimensionen. Trotz der o. a. Eingrenzungen und Kritik erlaubt die Taxonomie von Anderson und Krathwohl, einer Vielfalt an Contenttypen eine Struktur zu geben. Im Vordergrund soll hier die Analyse von Lernergebnissen stehen und nicht, in welchem Lernszenarium das Wissen erworben wurde (Mayer, Hertnagel \& Weber, 2009, S. 44).

\subsection{Flipped Classroom im Lehrrahmen von Bloom}

Bergmann und Sams (2014) beschreiben, wie diese Synergie von Ideen speziell mit dem umgekehrten Lernen (Flipped Learning) zusammenhängt. Sie haben das Konzept erweitert, um auszudrücken, wie jede einzelne Kategorie den Schüler*innen zugutekommen kann. Als Kritik kann hier angeführt werden, dass das umgekehrte Lernen nur altbewährte Praktiken guter Pädagog*innen sind, die neu verpackt und anders benannt sind. Forschungsergebnisse haben gezeigt, wie Menschen lernen und dabei drei Kategorien (Inhalt - Vertiefung des Lernens, Neugierde - weiter lernen, Beziehung - Verbindungen mit Studenten herstellen) nutzen, und regen damit die Diskussion weiter an, wie das umgekehrte Lernen Lehrenden und Lernenden auf effektive Weise helfen kann (Bergmann \& Sams, 2014, S. 29).

Die Taxonomie von Bloom ist ein Lehrrahmen, der häufig zur Diskussion von Lehren und Lernen verwendet wird. Obwohl es nicht der einzige Weg ist, die Art und Weise zu verstehen, wie Menschen lernen, ist diese Taxonomie ein guter Rahmen, um zu diskutieren, wie jede*r Einzelne den Lernprozess durchläuft (Bergmann \& Sams, 2014, S. 30). Eine Möglichkeit, die überarbeitete Version der Bloom-Taxonomie zu betrachten, besteht darin, am unteren Ende der Pyramide zu beginnen, die Grundlagen für das Lernen zu schaffen und die Schüler*innen zu höherwertigen Denkfähigkeiten (siehe Abbildung 2) zu führen. Die Pyramidendarstellung der Bloom-Taxonomie impliziert, dass Unterrichtszeit entsprechend verteilt werden sollte, wobei die Fertigkeiten niedrigerer Ordnung die meiste Zeit in Anspruch nehmen und die Fertigkeiten höherer Ordnung am wenigsten. Tatsächlich ist es das, was normalerweise im Klassenzimmer passiert: Lehrer*innen verbringen den Großteil ihrer Zeit mit Erinnerung 
und Verstand. Die Anwendung kommt zu kurz. Die Lehrer*innen erhoffen sich, dass sie zu höherwertigen Denkfähigkeiten kommen, aber für Analyse, Bewertung oder Kreativität wird nur sehr wenig Zeit eingeplant.

Bergmann und Sams glauben, dass von der Lehrerschaft erstellte Videos am besten als Hilfsmittel zur Vermittlung von Inhalten in den unteren beiden Stufen (Erinnern und Verstehen) der Bloom-Taxonomie dienen. So können die Lehrer*innen ihre wertvolle Unterrichtszeit mit den Schüler*innen verbringen, während diese sich an Aktivitäten beteiligen, die Fähigkeiten in den oberen Bereichen der Bloom-Taxonomie erfordern und die ein tieferes Lernen ermöglichen (Bergmann \& Sams, 2014, S. 30).

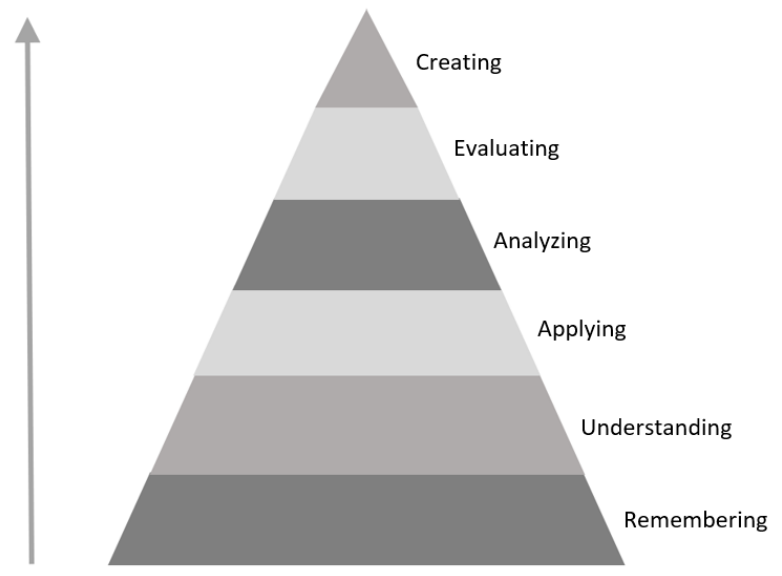

Abbildung 2: Inverted Bloom's taxonomy (eigene Darstellung nach Bergmann \& Sams, 2014, S. 31)

Was meinen Bergmann und Sams (2014) damit, die umgekehrte Klasse zu vertiefen? Wenn Lehrer*innen mit Hilfe des umgekehrten Lernens (Flipped Learning) tiefer in ein bestimmtes Thema eindringen, ermöglichen sie den Schüler*innen ein umfassenderes und gründlicheres Verständnis des Inhalts. Bei einem eher traditionellen Ansatz geschieht dies durch den sorgfältigen Aufbau des kognitiven Rahmens von der Basis der BloomTaxonomie aufwärts und es wird viel Zeit auf die Beherrschung der Inhalte verwendet. Die Schüler*innen lernen nach einem vorgeschriebenen Lehrplan, der sich höchstwahrscheinlich aus den gemeinsamen Kernzielen, den Bildungsstandards und dem professionellen Urteilsvermögen der Lehrenden ergibt. Die Schüler*innen müssen dann die Beherrschung des Inhalts in einer summativen Beurteilung nachweisen. Neu an dem umgekehrten Lernmodell ist die Art und Weise, wie der Inhalt vermittelt wird. Die Lehrkraft kann den asynchronen Unterricht per Video nutzen und ihre Schüler*innen mit Hilfe des umgekehrten Lernens tiefer in die Materie einführen (Bergmann \& Sams, 2014, S. 31).

Die Schüler*innen lernen nicht nur den erforderlichen Lehrplan, sondern sind aufgrund des oft durch umgekehrtes Lernen (Flipped Learning) ermöglichten Lernens auf höherer Ebene in der Lage, die Beherrschung von Themen zu demonstrieren, ihr neues Wissen auf einzigartige Situationen anzuwenden und Lernobjekte zu erstellen, die die Tiefe ihres Lernens demonstrieren (Bergmann \& Sams, 2014, S. 31).

Bergmann und Sams (2014, S. 33) stellten fest, dass die Auslagerung des unteren Endes der Bloom-Taxonomie aus dem Unterricht mehr Zeit für die Vertiefung des oberen Endes der Bloom-Taxonomie bietet. Daher soll Blooms Taxonomie von oben nach unten betrachtet werden (Abbildung 3). Die Taxonomie von Bloom soll erneut als Rahmenwerk verwendet, diesmal aber als eine umgekehrte Pyramide betrachtet werden.

Die Taxonomie von Bloom wurde in letzter Zeit in vielen Formen ausgedrückt, wobei diese umgekehrte Pyramide nur eine der vielen Vertauschungen ist. Dieser Top-down-Ansatz der Lernpyramide ist die Art und Weise, wie sich Untersuchungsstrategien an den Inhalt annähern (Bergmann \& Sams, 2014, S. 33). 


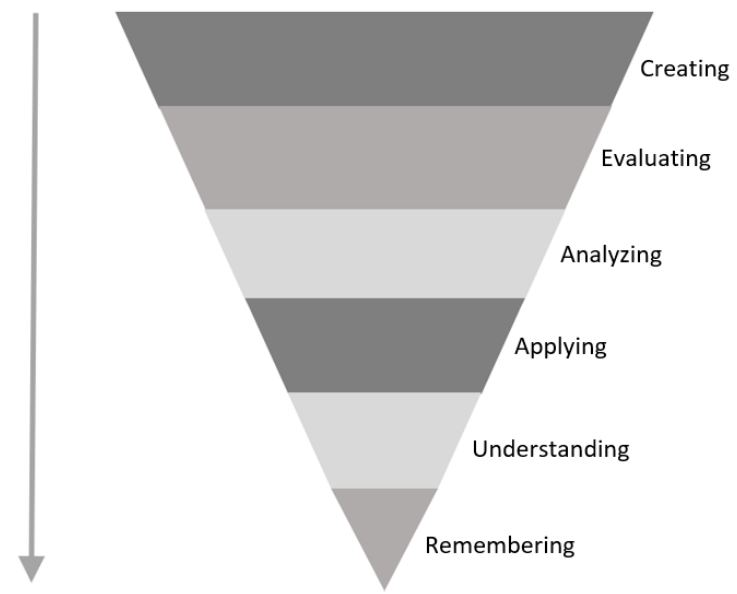

Abbildung 3: Inverted Bloom's taxonomy (eigene Darstellung nach Bergmann \& Sams, 2014, S. 34)

Die Hirnforschung weiß mittlerweile, dass das Gehirn einen methodischen Wechsel im Unterricht als Neugierde weckende Abwechslung sieht und zur Erhöhung der Aufmerksamkeit führt. Umfragen zufolge praktizieren nur zehn Prozent der Lehrer*innen Gruppenunterricht, obwohl man weiß, dass die Schüler*innen bei Frontalunterricht in einer 45-minütigen Schulstunde nur etwa 4,5 Minuten aufmerksam zuhören. Beim Arbeiten in Gruppen hingegen lernen sie, im Team zu arbeiten, selbstständig zu arbeiten und einfach mitzudenken. Der eklatanteste Vorteil ist, dass die Schüler*innen aktiv ins Lernen involviert sind, da sie anderen Gruppenmitgliedern Sachverhalte erklären, Stoff selbst erarbeiten und somit kreativer sind und selbst Verantwortung im Lernprozess übernehmen müssen. Effektiver Gruppenunterricht beinhaltet klare Aufgabenstellungen und das Präsentieren der Ergebnisse vor den anderen Mitschüler*innen (Korte, 2009, S. 295).

\section{Resümee und Ausblick}

Mit allen Erfahrungen und Dynamiken sowie allen negativen Erscheinungen zum Trotz wird die COVID-19Krise zu einem weiteren erheblichen Schritt zur kontinuierlichen Schulentwicklung im Hinblick auf den digitalen Wandel in der Schule beitragen. Aus der Sicht der Verfasserin wird es an vielen Schulen zur Einführung und Weiterentwicklung von Blended Learning kommen. Aus diesem Grund benötigt es noch mehr empirische Befunde zur optimalen Ausgestaltung der Kombination von Online- und Präsenz-Lehre an Schulen.

Flipped Classroom bietet eine Möglichkeit, die von Meyer (2020) postulierten didaktischen Ansprüche im Homeschooling umzusetzen. Das aufgebaute Arbeitsbündnis hat natürlich durch die Auslagerung von Lernsequenzen aus dem Unterricht Relevanz. Zentral scheint hier, dass Schüler*innen bereits Erfahrung im Umgang mit selbstreguliertem Lernen aus dem Präsenzunterricht mitbringen und diese dann im Homeschooling einsetzen können. Interessant für weitere Forschungsprojekte ist die Frage, inwieweit bzw. ob es überhaupt gelingen kann, Fähigkeiten im selbstgesteuerten Lernen erst im Homeschooling aufzubauen bzw. weiterzuentwickeln. Folgt man Meyers (2020) Anregungen weiter, so zeigt sich, dass Unterrichtseinheiten mit Flipped Classroom durch geeignetes Feedback an die Schüler*innen unterstützt bzw. basierend auf dem Feedback der Schüler*innen weiterentwickelt werden müssen. Die Methode des Flipped Classrooms bietet außerdem die Möglichkeit, bereits in der Selbsterarbeitungsphase jene Schüler*innen zu unterstützen, die ein Leistungsdefizit aufweisen. Dies scheint essentiell, da im Home Schooling die Gefahr besteht, dass sich die Schere zwischen leistungsstarken und leistungsschwachen Schüler*innen weiter vergrößert (Huber \& Helm, 2020, S. 56). 
Die Orientierung an Lernzieltaxonomien wie etwa an jenen von Bloom bei der Planung von Flipped Classroom stellt sicher, dass kognitiv und sozial aktivierende Aufgabenstellungen eingesetzt werden. Didaktisch bietet natürlich die Onlineumgebung hier viele Möglichkeiten, Aufgaben abseits von traditionellen Arbeitsblättern zu gestalten. Es muss natürlich beachtet werden, dass Flipped Classroom eine von vielen Methoden ist und die Entscheidung für eine Methode immer mit Hinblick auf Inhalt, Lehrziele und Zielgruppe getroffen werden muss. Im Sinne der Methodenvielfalt ist es sinnvoll, Flipped Classroom als eine von vielen Methoden im Unterricht einzusetzen.

Abschließend kann festgehalten werden, dass COVID-19 die Bildungswelt auf den Kopf gestellt und gezeigt hat, dass virtuelle Realitäten nicht nur zunehmen, sondern bereits da sind. Gezeigt hat sich weiters, dass Schule in dieser Zeit sowohl für einige Lehrer*innen als auch Schüler*innen in dieser Form sehr gut funktionierte, für andere allerdings nicht gänzlich. Es liegt nun an den Lehrenden, didaktische Konzepte, Unterrichtsmodelle sowie analoge und digitale, synchrone sowie asynchrone Unterrichtseinheiten, Präsenz- und Online-Lernen auch in Zukunft zu kombinieren, möglicherweise sogar gleichzeitig anzubieten, und die Transformation schulischen Lernens weiterzuführen und fortzusetzen.

\section{Literatur}

Baumgartner, P. (2011). Taxonomie von Unterrichtsmethoden - Ein Plädoyer für didaktische Vielfalt. Münster, New York, München, Berlin: Waxmann.

Bergmann, J. \& Sams, A. (2014). Flipped learning Gateway to student engagement. United States of America: International Society for Technology in Education.

Buchner, J. \& Schmid, S. (2019). Flipped Classroom Austria ... und der Unterricht steht kopf! Brunn am Gebirge: ikon VerlagsGesmbH.

Gruschka, A. (2007). „Was ist guter Unterricht?“. Über neue Allgemein-Modellierungen aus dem Geiste der empirischen Unterrichtsforschung. Pädagogische Korrespondenz (2007) 36, S. 10-43. https://pedocs.de/frontdoor.php?source_opus=7964

Hattie, J. (2013). Lernen sichtbar machen - überarbeitete deutschsprachige Ausgabe von „Visible Learning“ von Wolfgang Beywl und Klaus Zierer. Baltmannsweiler: Schneider Verlag Hohengehren GmbH.

Helmke, A. (2015). Unterrichtsqualität und Lehrerprofessionalität - Diagnose, Evaluation und Verbesserung des Unterrichts. Seelze-Velber: Kallmeyer mit Klett Friedrich Verlag GmbH

Huber, S. G., Günther, P. S., Schneider, N. Helm, C., Schwander, M., Schneider, J.A. \& Pruitt, J. (2020). COVID-19 - aktuelle Herausforderungen in Schule und Bildung. Münster: Waxmann Verlag GmbH. Zugriff am 2. April 2021 unter https://doi.org/10.31244/9783830942160

Huber, S. G. \& Helm, C. (2020). Lernen in Zeiten der Corona-Pandemie. Die Rolle familiärer Merkmale für das Lernen von Schüler*innen: Befunde vom Schul-Barometer in Deutschland, Österreich und der Schweiz. Die Deutsche Schule, Beiheft 16, S. 37-60.

Korte, M. (2009). Wie Kinder heute lernen - Was die Wissenschaft über das kindliche Gehirn weiß - Das Handbuch für den Schulerfolg. München: Deutsche Verlags-Anstalt.

Krathwohl, D., Bloom, B. \& Masia, B. (1975). Taxonomie von Lernzielen im affektiven Bereich. Weinheim und Basel: Beltz.

Mayer, H. O., Hertnagel, J. \& Weber, H. (2009). Lernzielüberprüfung im eLearning. München: Oldenbourg Wissenschaftsverlag $\mathrm{GmbH}$.

Mayr, K., Resinger, P. \& Schratz, M. (2009). E-Learning im Schulalltag. Bad Heilbrunn: Julius Klinkhardt Verlag.

Meyer, H. (2017). Was ist guter Unterricht? 15. Auflage. Berlin: Cornelson Verlag GmbH

Meyer, H. (2020). Didaktische Ansprüche an Homeschooling und Fernunterricht. Zugriff am 2. April 2021 unter https://unterrichten.digital/2020/05/07/hilbert-meyer-homeschooling/

Reinmann, G. (2005). Blended Learning in der Lehrerbildung - Grundlagen für die Konzeption innovativer Lernumgebungen. Lengerich: KM Druck.

Rustemeyer, R. (2011). Einführung in die Unterrichtspsychologie. Darmstadt: WBG Wissenschaftliche Buchgesellschaft. 
R\&E-SOURCE https://journal.ph-noe.ac.at

Schöftner, T. (2018). Möglichkeiten von webbasierten Online-Systemen zur Steigerung der Fremdsprachenkompetenz - Eine empirische Studie am Beispiel des Unterrichtsfaches Englisch. Hamburg: Dr. Kovac GmbH.

Spitzer, M. (2011). Lernen - Gehirnforschung und die Schule des Lebens. Heidelberg: Spektrum Akademischer Verlag.

Terhart, E. (1997). Lehr-Lern-Methoden. Weinheim und München: Juventa Verlag.

Unterrichten Digital (2021). Blended Learning - vom Corona-Chaos zur kontinuierlichen Schul- und Unterrichtsentwicklung. Zugriff am 2. April 2021 unter https://unterrichten.digital/2021/03/09/blended-learning-unterrichtschulentwicklung/\#Blended_Learning_als_didaktisches_Prinzip_fur_Distanzlernen

Werner, J., Ebel, C., Spannagel, C. \& Bayer, S. (2018) Flipped Classroom - Zeit für deinen Unterricht Praxisbeispiele, Erfahrungen und Handlungsempfehlungen. Gütersloh: Verlag Bertelsmann Stiftung.

Wiechmann, J. (1999). Zwölf Unterrichtsmethoden - Vielfalt für die Praxis. Weinheim und Basel: Beltz Verlag. 